\title{
NextMed, Augmented and Virtual Reality platform for 3D medical imaging visualization
}

\author{
Explanation of the software platform developed for 3D models visualization related with medical \\ images using Augmented and Virtual Reality technology.
}

\author{
Santiago González Izard \\ PhD Student \\ University of Salamanca, ARSOFT \\ Salamanca, Spain \\ santiago.gi@usal.es \\ Juan Antonio Juanes Méndez \\ Department of Anatomy \\ University of Salamanca \\ Salamanca, Spain \\ jajm@usal.es
}

\author{
Óscar Alonso Plaza \\ University of Salamanca \\ Salamanca, Spain \\ u157938@usal.es
}

\author{
Ramiro Sánchez Torres \\ University of Salamanca \\ Salamanca, Spain \\ ujhik@usal.es
}

\author{
Francisco José García-Peñalvo \\ University of Salamanca \\ Salamanca, Spain \\ fgarcia@usal.es
}

\begin{abstract}
The visualization of the radiological results with more advanced techniques than the current ones, such as Augmented Reality and Virtual Reality technologies, represent a great advance for medical professionals, by eliminating their imagination capacity as an indispensable requirement for the understanding of medical images. The problem is that for its application it is necessary to segment the anatomical areas of interest, and this currently involves the intervention of the human being. The Nextmed project is presented as a complete solution that includes DICOM images import, automatic segmentation of certain anatomical structures, 3D mesh generation of the segmented area, visualization engine with Augmented Reality and Virtual Reality, all thanks to different software platforms that have been implemented and detailed, including results obtained from real patients. We will focus on the visualization platform using both Augmented and Virtual Reality technologies to allow medical professionals to work with $3 \mathrm{~d}$ model representation of medical images in a different way taking advantage of new technologies.
\end{abstract}

\section{KEYWORDS}

Virtual Reality, Augmented Reality, Medical Imaging, Automatic Segmentation

\section{Introduction}

Medical imaging technology has undergone a constant evolution in recent decades. With the evolution of magnetic resonance imaging (MRI) or the introduction of new Computed Tomography (CT) techniques such as Multidetector Computed Tomography (MDCT), radiological diagnosis can be performed less invasively and more reliably [1,2]. There are several tools, like OsiriX or 3DSlicer [3,4], capable of producing high resolution $3 \mathrm{D}$ images from these medical images in just few seconds [5,6].

The problem is that manually segmentation, using tools such as those mentioned above, is complicated in many cases and too slow [7]. The automation of the 3D segmentation process of anatomical structures would be extremely convenient for doctors, radiologists or other professionals in the sector, since it would allow them to start working with threedimensional (3D) models instead of two-dimensional (2D) images, which is now expensive as it does not exist any system to automatically make the segmentation of anatomical structures [8]. 
The use of Augmented Reality (AR) and Virtual Reality (VR) technologies as more sophisticated visualization and interaction techniques facilitates manipulation and possible diagnosis from the $3 \mathrm{D}$ image $[9,10]$. These technologies are widely used for training purposes in medicine and other fields, but not for helping medical professionals in their daily work [11-15].

But before being able to visualize medical images taken from a CT, it is first necessary to segment the anatomical structure we would like to convert into a 3D model. We have created Computer Vision (CV) algorithms and Artificial Intelligence (AI) systems capable of automatic segmentation. This is one of the keys of this project, while the other would be the 3D visualization system, that allows not only to visualize the results of segmentation and 3D model creation modules, taking advantage of the three dimensions, but also to manipulate the 3D model and save images and notes for a possible future surgery. First of all, we need to understand DICOM format and the data this kind of files stores, highlighting those parameters that allow us to estimate Hounsfield Units (HU) [16,17].

In artificial vision there are various techniques that allow medical images segmentation. The problem is the variability between some patients and others and the variability between different scans. Hounsfield Units are used to partially reduce the difference between different CT scanning machines.

We can define the Hounsfield scale as a quantitative scale used in computed tomography studies to describe the different levels of radiodensity of human tissues.

The Hounsfield scale is the result of the transformation of the linear X-Ray attenuation coefficients scale into a new scale in which the attenuation value of distilled water in Normal Pressure and Temperature Conditions (CNPT) is defined as 0 Hounsfield Units, while the radiodensity of the air under Normal Pressure and Temperature Conditions (CNPT) is defined as $-1000 \mathrm{HU}$. The compact bone exceeds $1000 \mathrm{HU}$ values, and metal implants can exceed $2000 \mathrm{HU}$.

Therefore, the Hounsfield scale can facilitate a first phase in the segmentation, where we select voxels whose value on the Hounsfield scale coincides with the window of values associated with the anatomical structure to be segmented. However, a segmentation cannot be based just on this technique, since poorly defined anatomical structures are obtained and different organs or muscles may have overlapping values on the scale, resulting in segmentation errors. This is why it is necessary to implement specific Computer Vision algorithms or follow Artificial Intelligence techniques to achieve satisfactory automatic segmentation results.

Using VTK and ITK libraries [18-22], we have created computer vision algorithms for automatic segmentation of lungs, hearts, arteries and veins inside lung, tracheas and spines. This is a great advance respecting previous versions of this project [23,24]. New algorithms for automatic segmentation of more anatomical structures are being designed, so in the future we will be able to automatically separate the main anatomical structures available in a CT scan. We are working with different specialists of the University Hospital of Salamanca to choose the most important anatomical structures. Images obtained with Magnetic Resonance (MR) scans will be also compatible in the future.

Once we get automatic segmentation, hospitals can start using our system to be able to work with medical imaging using new technologies, being able to print the lung of a patient or visualize it with Augmented or Virtual Reality. But with previous steps we still don't have the 3D model, we just have a series of images where only the lung appears, but we need to convert this into a $3 \mathrm{D}$ model. We have to continue with the process.

Applying different algorithms, we get a 3D mesh that can be imported into our visualization platform, created using the Unity3D game engine [25-29], that takes advantage of AR and VR to work with the 3D model.

In this article we will focus on the Augmented and Virtual Reality Visualization Platform. However, one of the keys of this system is the automatic segmentation module, so we will begin by describing the DICOM format for medical imaging, the input point for this module. Then software used to create computer vision algorithms will be described, but not the algorithms itself, as this is really extensive and other articles will be published about this subject. We continue explaining how we have implemented the visualization platform, from the Augmented and Virtual Reality system to the cloud platform needed to store and manage all data. After this, the results are described showing some images of the 3D models automatically obtained from DICOM data, visualized using both the Augmented Reality and the Virtual Reality platform. We finish with a discussion and conclusion section about how current and future technology will change the way medical professionals work with medical imaging.

This article is a continuation of the work of this team on this project, described in previous articles [23,24]. The project, entitled Nextmed, has more advanced source code regarding previous versions, with a computer vision module able to automatically generate 3D models of different anatomical areas of the human body, tested with more than 1000 CT scans. Also, the Augmented and Virtual Reality platform is now connected with the computer vision module and an upload module has been designed to be able to upload DICOM to the cloud platform. The VR system has also been adapted to work with new headset models. So Nextmed can now be used in a real medical environment to upload DICOM, automatically segment some anatomical structures, generate the $3 \mathrm{D}$ mesh and download those $3 \mathrm{D}$ meshes to the AR and VR visualization platform to be able to study and manipulate them. So, this is the first version that can be installed in a hospital to start with the first tests on real environments. 


\section{Material and Method}

\subsection{Understanding DICOM}

The first input our system receives is DICOM dataset obtained from a CT scan. We have used CT scans obtained by the University Hospital of Salamanca and public online datasets such us LIDC-IDRI [30], with 1018 CT scans of 1010 patients.

The information is stored in each DICOM file in a series of data elements, each of which has a label to recognize it (TAG), a data type (Value Representation), a value length (Value Length) and the stored information (Value Field), as can be seen in Figure 1.

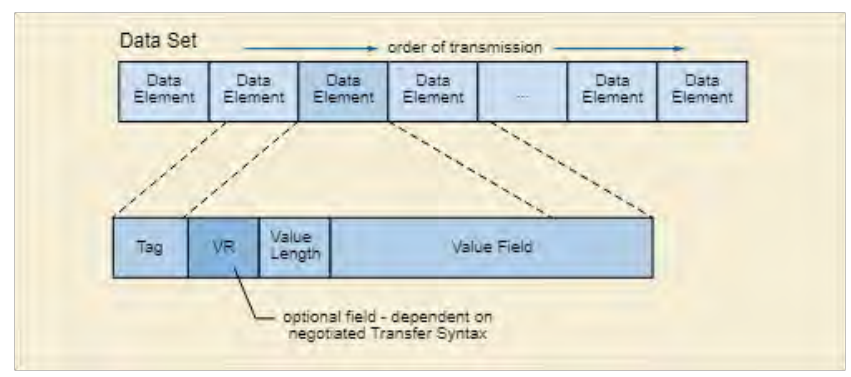

Figure 1: Value Types stored in DICOM file

The generic fields required by the standard used to develop the project have been: Pixel spacing, slice Thickness, Image Position, Image Orientation, Image Pixel, Photometric Interpretation, Rows, Columns, Bits Allocated, Bits Stored, Pixel Aspect Ratio, Pixel Data.

The DICOM format presents several specific fields for CT. We use: Rescale Type, Rescale Intercept and Rescale Slope. With these three fields, we can calculate Hounsfield Units (HU):

$$
H U=1000 \times \frac{\mu_{t}-\mu_{\text {agua }}}{\mu_{\text {agua }}}
$$

$\mathrm{HU}$ is very important as it is used for the first filters applied by our computer vision algorithms.

\subsection{Computer Vision Algorithms}

The ITK and VTK libraries have been used to implement automatic segmentation algorithms.

ITK is an open source library specializing in the treatment of medical images, with support for DICOM images.

VTK is an open source library specialized in medical image visualization. It has been developed by Kitware, just like ITK. It presents visualization algorithms with special techniques for the treatment of voxels such as the use of color maps depending on the intensities, transparency or automatic adjustment of the image quality to maintain constant FPS (Frames Per Second).

Both ITK and VTK have been used to create custom algorithms for each anatomical structure we are now able to segment automatically. For the implementation of these algorithms we have used not only HU, but also complex morphological studies based on Computer Vision, taking into account that each patient can present the same parts of the body in different ways. We got more than $90 \%$ of success in the segmentation of lungs, spines and other anatomical structures, and we continue working on these algorithms to improve the percent and to be able to automatically segment new anatomical areas based on the interest of the medical community.

\subsection{AR and VR Platform}

Unity3D (commonly called Unity) is a powerful multiplatform video game engine used in a large number of video games created around the world. It also offers a powerful platform for creating applications oriented to industry, education and, of course, medicine.

Unity3D has been chosen for the design of our application due to its powerful 3D rendering engine, its support for $A R$ and VR technologies and its integrated cross-platform system.

On the other hand, Vuforia is currently one of the most used Augmented Reality SDKs, due to its artificial vision technology, marker tracking and its support for various platforms [31,32].

Due to all these features, Vuforia is presented as the most complete solution at the moment when implementing our augmented reality display functions. However, the use of Vuforia corresponds only to the first phase of development, since the entire Augmented Reality module will be adapted to use Arcore, Google native Augmented Reality SDK. The option of using the Magic Leap SDK is also being considered, in order to take advantage of the potential offered by the hardware recently launched by this company, and that promises a breakthrough in terms of the concept of Mixed Reality, facilitating placement in the operating room of previously generated virtual elements: from the radiological images to the 3D models generated or the results obtained from the preoperative study.

For the development of the application in Virtual Reality we have chosen the development kits provided by Oculus VR and HTC Vive. In both cases, these are powerful solutions in the VR industry with highly tested systems in the market.

Oculus GO has been chosen for its lightness, versatility and immediacy when accessing the contents, which allows the doctor to visualize the models using this technology without requiring a pre-established or restrictive space [33].

However, HTC Vive offers a deeper interaction and greater image resolution, which enables its use in the scientific field allowing greater fidelity when making a diagnosis [34]. In addition, the fact that HTC Vive glasses are connected to a computer increases the processing and rendering power, so you can work with 3D models with a higher number of polygons and with volumes with a greater number of voxels, increasing the quality of 3D medical images.

Having checked the advantages and disadvantages of these two technologies it has been decided to take both solutions into account, since on the one hand HTC Vive has the advantage of being able to render very heavy models, while Oculus Go has the advantage of being an economic standalone device and easily transportable. On the other hand, Oculus recently launched a new standalone device, named Oculus Quest. In this version of the system we have included compatibility for both HTC Vive 
and Oculus Quest, as it is necessary to adapt the software to the new hardware.

Among the different features offered by the two viewing platforms (AR and VR) we find a shader-based cutting system that provides the possibility of making several sections on the model while it is not necessary to modify it, what allows the user to undo the last cuts, using for this a reduced processing time compared to previous versions of the system. This is achieved by generating cutting planes that the shader takes into account when choosing which points of the model to render and which not. For each point of the model the shader checks if it is located in the space defined by the cutting planes. The program code of a shader is executed once per pixel of our image, which is why optimization is critical to achieve proper operation. In previous versions the implementation of the model cut was carried out by generating two new models, crossing the vertex matrices that make up the model and generating two different matrices. This meant a very high processing time and incorrect results for certain 3D models.

To improve efficiency, the only part that runs continuously during execution are the pixel rendering functions (thus ignoring the reloading of properties and variables).

Shaders are algorithms that define the final appearance of each pixel based on their material and the lighting they receive. They are therefore an important part of the Unity rendering process, along with the materials and textures.

\subsection{Software Architecture}

Nextmed is composed of three subsystems that share much of the source code: An Augmented Reality system, another Virtual Reality system and finally another for PC. The use of Model View Controller (MVC) pattern (figure 2) allows to perfectly differentiate the code associated with the data, the one related to all the main logic and finally another module for the view. In figure 2 we can see how the different scripts are organized in the project and how they interact. In the case of the data module, it practically does not change between the three subsystems. The view generates the same interfaces in all three cases, however, the interaction is different in each of them, so both the view and the controller are affected by this variability of interaction and presentation of the data. To facilitate the understanding of the diagram, not all the variants of the scripts have been shown in figure 2 .

As a result of the model management, the Factory Method design pattern has been used to define two types of data access. SQLDaoFactory creates the data access interfaces (DAO) with access to the relational database. PersistentDAOFactory does the same for local storage. Saving data locally and on the cloud allows the users to access data from any device: if data is present locally then it will not be necessary to download them, if not then the system will try to download all data from the cloud platform.

Both the architecture and the functionality are very similar for all implementations in different technologies, since all versions share the same modules except the one oriented to the rendering of the $3 \mathrm{D}$ model, which of course is different depending on whether it is the PC version, Augmented Reality or Virtual Reality.

All scripts have been implemented using C\# language. This is the most used language in Unity3D for programming. On the other hand, Java language has been used for programming the Cloud Platform, configuring a Linux server to install all the programs.

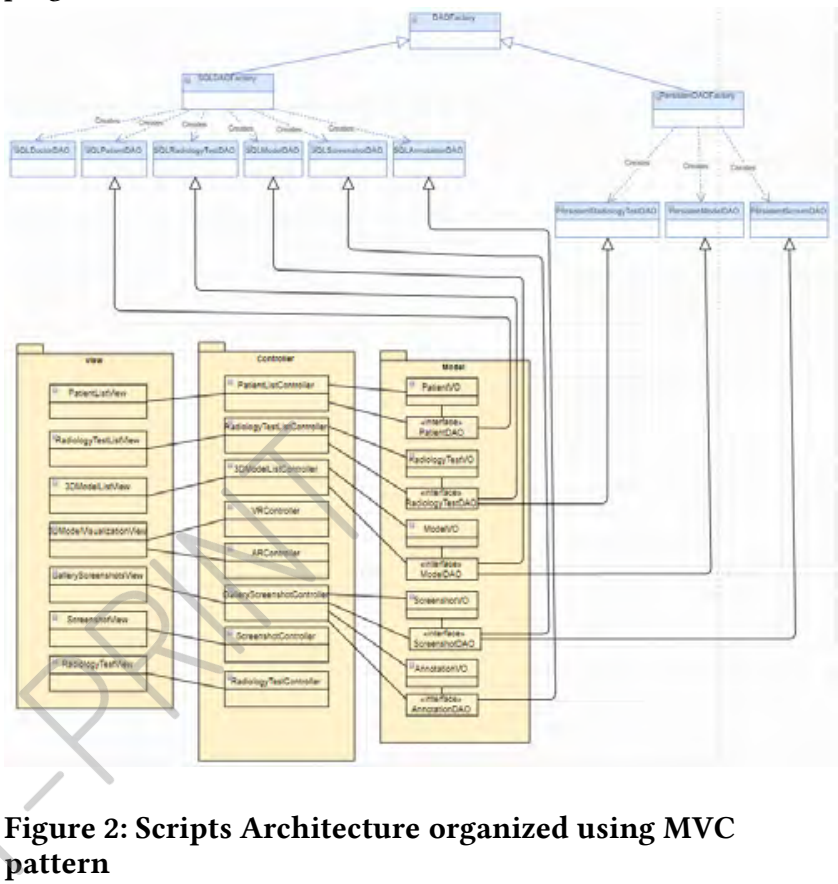

\subsection{Cloud Platform}

All data accessible by our application are currently stored on a Cloud server through a file structure that sorts the generated models, the DICOMs received by the system and the captures made and notes taken. The routes related to these files, with the data of patients and doctors are referenced by a relational database.

Java has been used for REST Web Services implementation, which allow the application to interact with the database. These are included in the Tomcat servlet container and perform the functions of managing queries, insertions and modifications in the MySQL database.

The diagram represented in the Figure 3 shows the data distribution in the database and the file hierarchy used for files storage. 


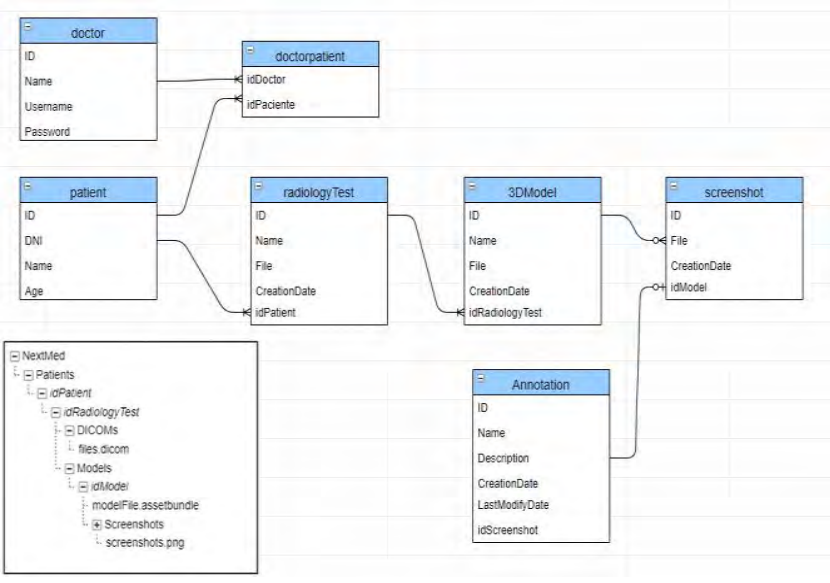

Figure 3: Database tables and files hierarchy in cloud server

\section{Results}

Taking as reference the results obtained when applying the artificial vision algorithms for automatic segmentation, 98\% of success stories for lung segmentation, $95 \%$ for vertebral columns, $98 \%$ for arteries and veins in lung and $65 \%$ have been achieved for the segmentation of hearts (improved in future phases).

In the images presented in Figures 4-7, we can see the result of the $3 \mathrm{D}$ visualization of the different segmented anatomical structures.

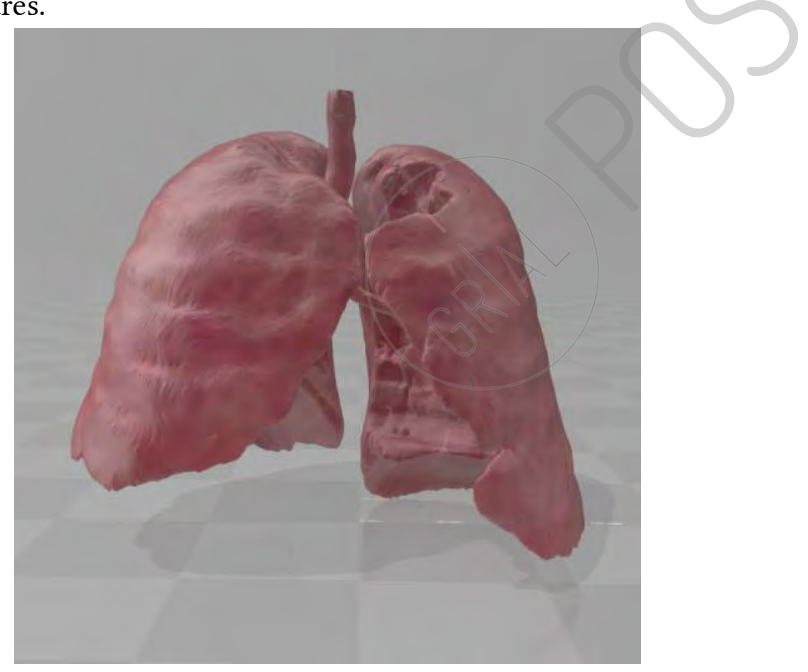

Figure 4: 3D model representation for automatically segmented lung

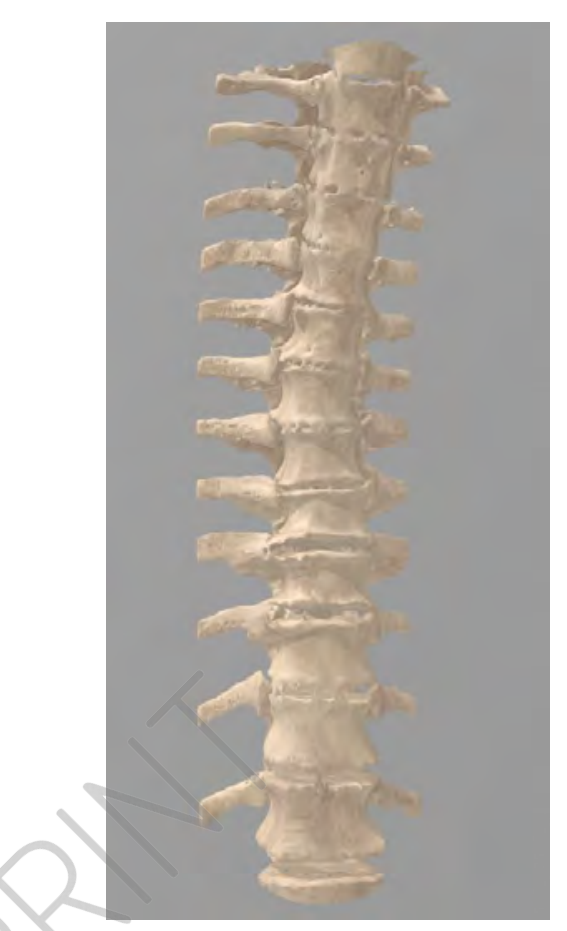

Figure 5: Automatically segmented spine

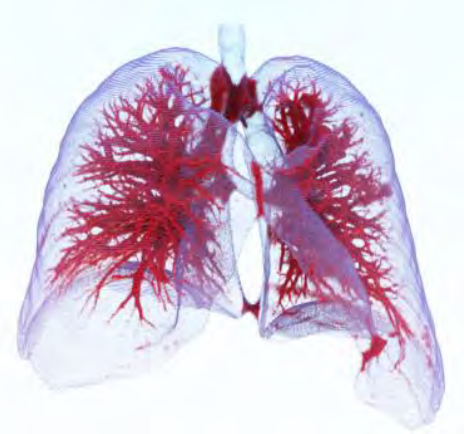

Figure 6: Automatically segmented veins and arteries in lung

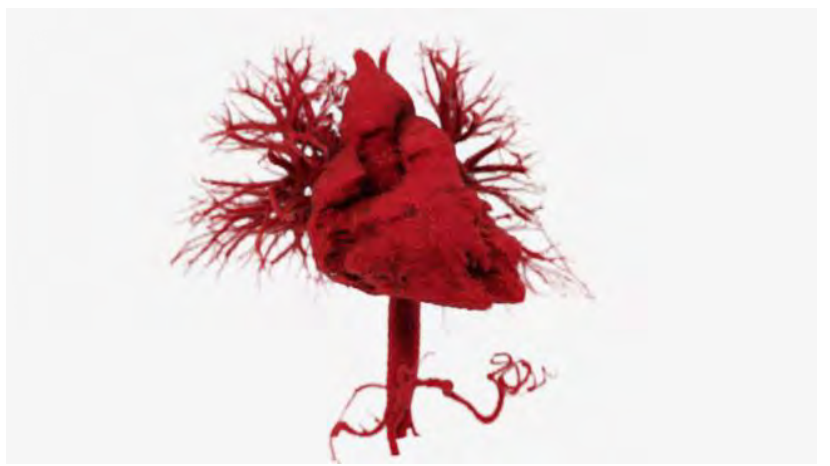

Figure 7: Automatically segmented heart 


\subsection{AR Platform}

For the visualization and manipulation with Augmented Reality of the 3D model produced from medical images, the multiplatform application NextMed AR has been designed. Built on Unity and using Vuforia technology, it provides an easily usable environment for the medical world in which the consultation and diagnosis on the 3D model generated from the radiological results can be carried out easily.

The NextMed AR application has been implemented for Android and iOS devices in this iteration of the project, improving many functionalities respecting previous versions, such as the cutting module. The next step is to implement our system for Augmented Reality glasses like the Magic Leap One or Microsoft Holo Lens. With this technology we can place the virtual elements of the system in the real world and achieve a lighter interaction using our own hands. This implies that the surgeon may be working in the operating room with glasses and place, simply with manual gestures, different contents around the patient: the 3D model of the anatomical area undergoing surgery, medical images, results of the pre-surgical study carried out... This interaction capacity is very important to avoid any kind of contamination, as it is not necessary to manipulate any computer or tablet. In addition, to visualize the different virtual elements you simply have to move your head and look to the area where you have previously placed them.

In Nextmed, the models are classified in the system by patient and by radiological test to provide an orderly and intuitive consultation. The authorized doctor will be able to access the models of his patients through a personal account. This will make the registration through a web application that is responsible for all user management and data loading.

In Figure 8 we can see that the medical professional, who has previously logged in, has accessed the results of the patient "Juan de la Hoz" (we do not use real names), who has a single radiological test (Test 1 ) and which in turn contains a single generated 3D model For those medical images. This 3D model (Lung) can be visualized both with Augmented Reality, and with Virtual Reality, as well as being able to access the gallery of images that have been taken for that model. Each of these images may be accompanied by an explanation that the medical professional can take as a result of a pre-surgical study. As explained above, the objective is that all this information can later be easily accessible during surgery. Finally, we appreciate in the image a visualization with Augmented Reality of the 3D model.
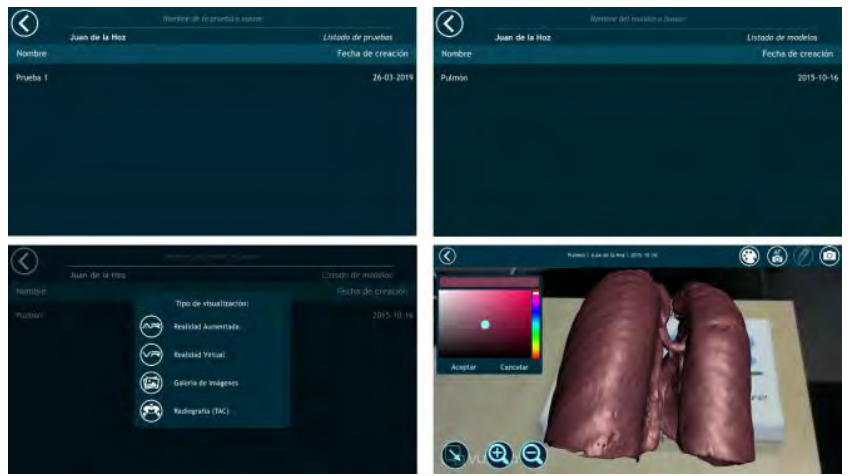

Figure 8: Different interfaces of AR visualization system

In order to carry out an extensive analysis of the model obtained automatically from the medical images; it is necessary to implement the cutting tool (Figure 9). In this way we can study its interior and isolate areas of interest.
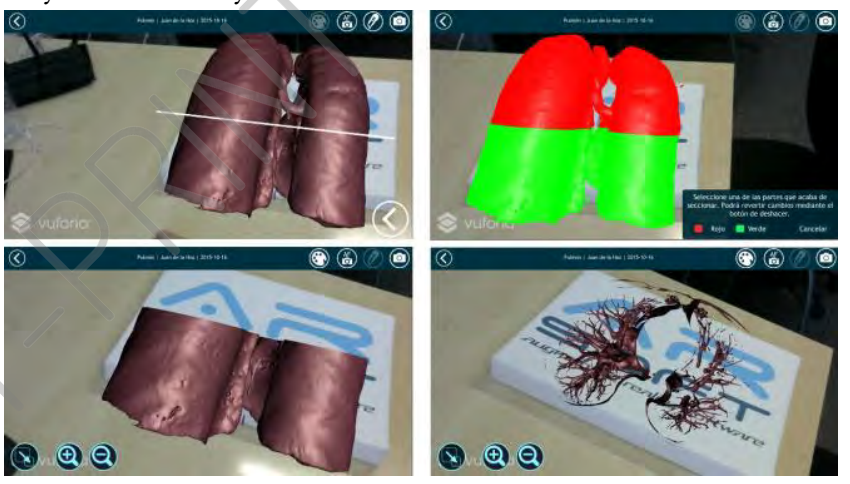

Figure 9: Screenshots showing cutting functionality for lung and veins and arteries

The cutting process is based on a versatile cutting system that allows multiple cutting of 3D models in different planes, making it possible to undo the changes of the different cuts made efficiently thanks to a new implementation of this
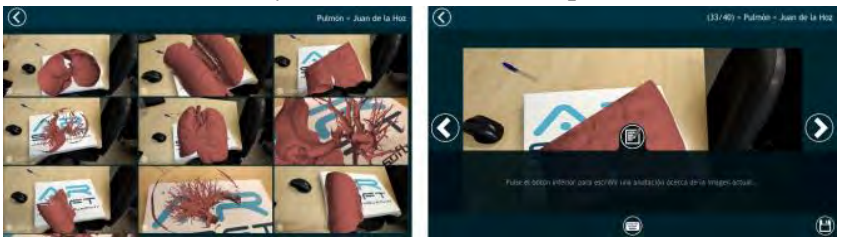

Figure 10: Surgery planification module for taking screenshots and notes

\subsection{VR Platform}

The NextMed VR version harnesses the power of user interaction and immersion provided by Virtual Reality technology (figure 11). In this way the manipulation of the model becomes easier and intuitive. This can be very important to facilitate a diagnosis, as the professional can move around the model and visualize it with more detail. In addition, in future 
versions the medical professional may even be introduced into the 3D model to study it as if "walking inside".

In the first iterations of the project we have been using Oculus Go headsets, with the aim of being able to perform tests in hospitals with a minor investment from them. In this version, it has been implemented for more powerful VR technologies, getting better image quality and interaction, and facilitating a correct and more accurate diagnosis. Specifically, we are now able to use Oculus Quest glasses, which as explained before providing greater processing and rendering potential.

The cutting process (Figure 11) uses the same algorithm as the AR version, although by taking advantage of VR technology we can make more thorough analyzes of the cut areas. With this we can approach everything we want to the model and carefully study areas that are considered of greatest interest for diagnosis. Keep in mind that the following images show what we can see with stereoscopic glasses, taking into account that thanks to the motion sensors present in glasses such as the Oculus Quest, we can walk around it. All these options provide a capacity to study medical images that so far is not achieved with any other system, thanks to AR and VR.

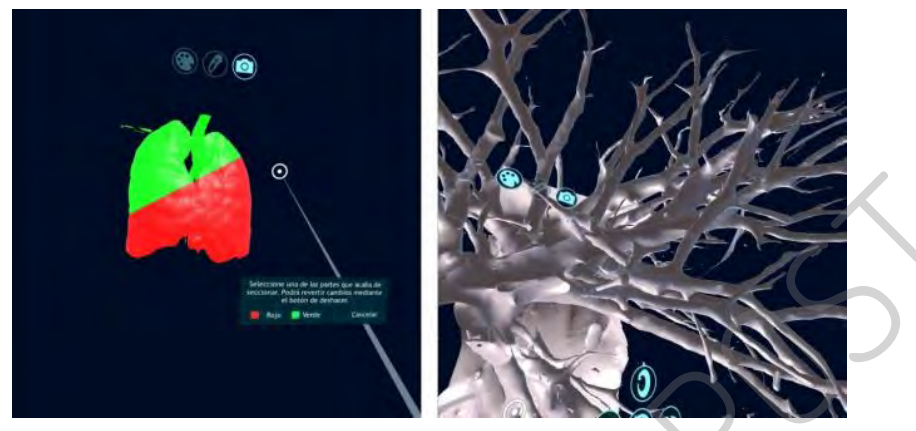

Figure 11: Medical Image 3D visualization with VR system

\section{Discussion}

The power of the 3D viewer of tools such as Osirix or 3D Slicer, as well as similar ones, is unquestionable, and they are two important tools in the medical industry. On the other hand, the tool presented in this article aims to take advantage of this quality of visualization offered by the VTK and ITK libraries, the same ones that OsiriX and 3DSlicer use internally, and also complete it with the advantages that Virtual and Augmented Reality technologies provide, such as interaction in a 3D space and realistic visualization of the results.

Augmented Reality is a technology that is currently being widely implemented in some sectors, highlighting the industrial sector. In the next 5 years its expansion will be directed to other sectors, and one of them will undoubtedly be the medical one. In addition, the advance in the hardware used by the AR will cause more and more people to start using Augmented Reality glasses, or even contact lenses (the latter presumably by the end of the next decade). The democratization of technology will mean the complete expansion of Augmented Reality in all sectors of our society, and the health sector will be one of the most benefited from it, due to the large number of advantages it brings to medical professionals.

Visualizing medical results as 3D models will not only be attractive, it will be necessary from the point of view of professionals, who will have powerful physical tools to visualize and work with them in a three-dimensional space, such us mixed reality glasses. This will greatly eliminate the current need to have an imagination capacity to mentally build the anatomical structures of the 3D patient based on the visualization of twodimensional medical images. In fact, this is currently a problem for many specialists and even radiologists.

Results obtained in this work demonstrate AR and VR can be very useful for medical professionals when analyzing medical imaging, as they don't need to build a mental reconstruction of the images. Also, being able to access the 3D model reconstruction, notes, images and more during the surgery can be a very interesting tool. In future developments, the 3D model can be created on the fly, which means being able to create a CT during surgery to have at the moment the segmented 3D model of the patient including, for example, visualization of the medical surgical instrumentation. By now, we allow radiologists and specialists to view the radiological results with the lasts technologies and get a pre-surgical study that can be easily accessed during surgery. All this can be done at low cost, as human action is not necessary thanks to an automated process based on computer vision algorithms specifically designed to segment certain parts of the human body, including bones, muscles, organs, arteries and veins.

In relation with previous versions of this system [23,24], automatic segmentation module can now generate the 3D mesh as an OBJ format file. Also, computer vision algorithms are now more accurate and new anatomical structures can be automatically segmented. The continuation of the work on this project will allow to segment new areas, new functionalities will be included in the visualization platforms and compatibility with new innovative devices such us Magic Leap mixed reality glasses will be possible. This device will be very useful while visualizing and analyzing the 3D model, as their tracking system and the rendering powerful will allow the development of very interesting new functionalities.

On the oher hand, Artificial Intelligence will change the diagnostic methodology in the next few years, making it easier for medical professionals to analyze the radiological results and improving the visualization techniques so far. In the next phase of this project, Artificial Intelligence will be used to support artificial vision algorithms during automatic segmentation, and later, in more advanced phases, the potential of AI could be exploited to begin working with techniques of automatic diagnosis of diseases, the results of which must be monitored, at least with the first systems of this type. AI community is currently working on this, so we have news about this very soon with successfully results. 


\section{Conclusions}

The work done so far in the Nextmed project, represents a qualitative leap in terms of the study of medical imaging, mainly due to two important points. First, we introduce the possibility of the use of automatic segmentation in the daily work of medical professionals. This is being done by some workstations, but they do not allow to export the 3D model as a mesh to be able to print it or visualize it out of the workstation, and manual work must usually be done. Secondly, we allow the visualization and manipulation of 3D models in an industrialized way by means of Augmented Reality and Virtual Reality technologies. So far, there have been numerous cases in which systems are designed to visualize a specific 3D model with these techniques, however the future of these technologies in the medical sector is not just for specific cases, but in being usable for all radiological results performed on patients, so when the specialist get the medical images it also receives a segmented 3D model. That is precisely the objective of Nextmed: to bring the potential of Augmented and Virtual Reality to all hospitals, and at this time we already have all the tools to do so, increasing the amount of anatomical structures generated automatically with each new advance in the project, and also with new functionalities.

In the future, doctors and radiologists will no longer have to study medical images using light beams: Augmented Reality and Virtual Reality will be used instead. The truth is that in the last decades there has not been much progress in the visualization of medical imaging. There have been many advances in the power of radiodiagnostic machines, image analysis software and available workstations, but there has been no disruptive change that takes advantage of the latest technologies that emerged during the 21st century. The Nextmed project aims to be that disruptive change, which allows professionals to work with medical images taking advantage of the technologies of Artificial Vision, Artificial Intelligence, Augmented Reality and Virtual Reality without the need for heavy periods of training and transition or large economic investments.

Next steps for this project will include the installation of this version in a real hospital to be used with current patients. In the following publications we will be able to publish the results of these tests. Although we have been testing the automatic segmentation algorithms with real CT scans, feedback obtained from medical professionals using Nextmed system will be very useful for next versions.

\section{ACKNOWLEDGMENTS}

The authors would like to specifically thank the members of ARSOFT, company specialized in Virtual and Augmented Reality systems and located in the Science Park of the University of Salamanca. This research work has been carried out within Education in Knowledge Society $\mathrm{PhD}$ Programme of the University of Salamanca $[35,36]$. This project and researches have been funded by the Challenges-Collaboration program of the European Union, with file number RTC-2017-6682-1.

\section{REFERENCES}

[1] Kido T, Kurata A, Higashino H, Sugawara Y, Okayama H, Higaki J, Anno H, Katada K, Mori S, Tanada S, Endo M, Mochizuki T (2007) Cardiac imaging using 256-detector row four-dimensional CT: preliminary clinical report. Radiat Med 25: 38-44

[2] Meaney J, Goyen M (2007) Recent advances in contrast-enhanced magnetic resonance angiography. Eur Radiol 17(Suppl 2): B2-B6.

[3] Osman Ratib, Antoine Rosset (2006). Open-source software in medical imaging: development of OsiriX. DOI 10.1007/s11548-006-0056-2

[4] Adriaan, Germain (2011-08-16). 3dslicer. Brev Publishing. ISBN 9786136666464.

[5] Doi K (2006) Diagnostic imaging over the last 50 years: research and development in medical imaging science and technology. Phys Med Biol 51: R5-R27

[6] Kirchgeorg M, Prokop M (1998) Increasing spiral CT benefits with postprocessing applications. Eur J Radiol 28: 39-54

[7] A. Hill, A. Thornham and C. J. Taylor (1997). Model-Based Interpretation of 3D Medical Images

[8] Neeraj Sharma and Lalit M. Aggarwal (2010) Automated medical image segmentation techniques

[9] Jeffrey H. Schuhaiber (2004) Augmented Reality in Surgery

[10] BMJ 2001;323:912

[11] Santiago González Izard Juan Antonio Juanes Méndez, Pablo Ruisoto Palomera. Virtual Reality Educational Tool for Human Anatomy. J Med Syst (2017) 41: 76. https://doi.org/10.1007/s10916-017-0723-6

[12] Santiago González Izard, Juan Antonio Juanes Méndez, Francisco José García Peñalvo, Jesús $M^{a}$ Gonçalvez Estella, $M^{a}$ José Sánchez Ledesma, Pablo Ruisoto Palomera. Virtual Reality as an Educational and Training Tool for Medicine. J Med Syst (2018) 42: 50. https://doi.org/10.1007/s10916-018-0900-2

[13] Santiago González Izard and Juan Antonio Juanes Méndez. 2016. Virtual reality medical training system. In Proceedings of the Fourth International Conference on Technological Ecosystems for Enhancing Multiculturality (TEEM '16), Francisco José García-Peñalvo (Ed.). ACM, New York, NY, USA 479-485. DOI: https://doi.org/10.1145/3012430.3012560

[14] Santiago González Izard, Juan A. Juanes Méndez, Francisco J. García-Peñalvo, Marcelo Jiménez López, Francisco Pastor Vázquez, and Pablo Ruisoto. 2017 $360^{\circ}$ vision applications for medical training. In Proceedings of the 5th International Conference on Technological Ecosystems for Enhancing Multiculturality (TEEM 2017), Juan Manuel Dodero, María Soledad Ibarra Sáiz, and Iván Ruiz Rube (Eds.). ACM, New York, NY, USA, Article 55, 7 pages. DOI: https://doi.org/10.1145/3144826.3145405

[15] Santiago González Izard, Juan Antonio Juanes Méndez, Jesús $M^{a}$ Gonçalvez Estella, $M^{a}$ José Sánchez Ledesma, Francisco J. García-Peñalvo, and Pablo Ruisoto. 2017. Virtual Simulation for Scoliosis Surgery. In Proceedings of the 5th International Conference on Technological Ecosystems for Enhancing Multiculturality (TEEM 2017), Juan Manuel Dodero, María Soledad Ibarra Sáiz, and Iván Ruiz Rube (Eds.). ACM, New York, NY, USA, Article 54, 8 pages. DOI: https://doi.org/10.1145/3144826.3145404

[16] Larobina, M., \& Murino, L. (2014). Medical image file formats. Journal of digital imaging, 27(2), 200-206.

[17] http://dicom.nema.org/medical/dicom/current/output/html/part05.html

[18] Wheeler, G., Deng, S., Toussaint, N., Pushparajah, K., Schnabel, J., Simpson, J., Gomez, A. 2018. Virtual Interaction and Visualisation of 3D Medical Imaging Data with VTK and Unity, Healthcare Technology Letters, DOI: 10.1049/htl.2018.5064

[19] Schroeder, W. J., Avila, L. S., \& Hoffman, W. (2000). Visualizing with VTK: tutorial. IEEE Computer graphics and applications, 20(5), 20-27.

[20] Geveci, B., Schroeder, W., Brown, A., \& Wilson, G. (2012). VTK. The Architecture of Open Source Applications, 1, 387-402.

[21] Schroeder, W., Ng, L., \& Cates, J. (2003). The ITK software guide.

[22] Yoo, T. S., Ackerman, M. J., Lorensen, W. E., Schroeder, W., Chalana, V., Aylward, S., ... \& Whitaker, R. (2002). Engineering and algorithm design for an image processing API: a technical report on ITK-the insight toolkit. Studies in health technology and informatics, 586-592.

[23] Santiago González Izard, Juan Antonio Juanes Méndez, Pablo Ruisoto Palomera, Francisco J. García-Peñalvo. Applications of Virtual and Augmented Reality in Biomedical Imaging. J Med Syst (2019) 43: 102 https://doi.org/10.1007/s10916-019-1239-z 
[24] Santiago González Izard, Juan A. Juanes Méndez, Pablo Ruisoto, and Francisco José García-Peñalvo. 2018. NextMed: How to enhance 3D radiological images with Augmented and Virtual Reality. In Proceedings of the Sixth International Conference on Technological Ecosystems for Enhancing Multiculturality (TEEM'18), Francisco José García-Peñalvo (Ed.). ACM, New York, NY, USA, 397-404. DOI: https://doi.org/10.1145/3284179.3284247

[25] RH Creighton (2010). Unity 3D game development by example: A Seat-ofyour-pants manual for building fun, groovy Little games quickly

[26] S. L. Kim, H. J. Suk, J. H. Kang, J. M. Jung, T. H. Laine and J. Westlin, "Using Unity 3D to facilitate mobile augmented reality game development," 2014 IEEE World Forum on Internet of Things (WF-IoT), Seoul, 2014, pp. 21-26. DOI: 10.1109/WF-IoT.2014.6803110

[27] J. Xie, "Research on key technologies base Unity3D game engine," 2012 7th International Conference on Computer Science \& Education (ICCSE), Melbourne, VIC, 2012, pp. 695-699. DOI: 10.1109/ICCSE.2012.6295169

[28] S. Wang, Z. Mao, C. Zeng, H. Gong, S. Li and B. Chen, "A new method of virtual reality based on Unity3D," 2010 18th International Conference on Geoinformatics, Beijing, 2010, pp. 1-5. DOI: 10.1109/GEOINFORMATICS.2010.5567608

[29] F. Messaoudi, G. Simon and A. Ksentini, "Dissecting games engines: The case of Unity3D," 2015 International Workshop on Network and Systems Support for Games (NetGames), Zagreb, 2015, pp. 1-6. DOI: 10.1109/NetGames.2015.7382990

[30] Armato III, Samuel G., McLennan, Geoffrey, Bidaut, Luc, McNitt-Gray, Michael F., Meyer, Charles R., Reeves, Anthony P., ... Clarke, Laurence P. (2015). Data From LIDC-IDRI The Cancer Imaging Archive. http://doi.org/10.7937/K9/TCIA.2015.LO9QL9SX

[31] Vuforia. 2019. PTC augmented-reality. [ONLINE] Available at https://www.ptc.com/en/products/augmented-reality. [Accessed 9 May 2019].

[32] Alexandro Simonetti Ibañez, Josep Paredes Figueras (2013). Vuforia v1.5 SDK: Analysis and evaluation of capabilities

[33] Parth Rajesh Desai, Pooja Nikhil Desai, Komal Deepak Ajmera, Khushbu Mehta (2014). A review Paper on Oculus Rift-A Virtual Reality Headset

[34] Diederick C. Niehorster, Li Li, Markus Lappe (2017) The Accuracy and Precision of Position and Orientation Tracking in the HTC Vive Virtual Reality System for Scientific Research.

[35] F. J. García-Peñalvo. 2014. Formación en la sociedad del conocimiento, un programa de doctorado con una perspectiva interdisciplinar. Education in the Knowledge Society 15, 1, 4-9

[36] F. J. García-Peñalvo. 2015. Engineering contributions to a Knowledge Society multicultural perspective. IEEE Revista Iberoamericana de Tecnologías del Aprendizaje (IEEE RITA) 10, 1, 17-18. DOI:10.1109/RITA.2015.2391371 